\title{
Autoimmunity to annexin A2 predicts mortality among hospitalised COVID-19 patients
}

\author{
Copyright @The authors 2021. \\ This version is distributed under \\ the terms of the Creative \\ Commons Attribution \\ Non-Commercial Licence 4.0. \\ For commercial reproduction \\ rights and permissions contact \\ permissions@ersnet.org \\ Received: 15 Jan 2021 \\ Accepted: 24 June 2021
}

\section{○ @®丹}

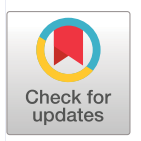

\section{To the Editor:}

The pathophysiology of severe coronavirus disease 2019 (COVID-19) has largely been attributed to a hyper-inflammatory response without a clear indication of the underlying mechanism [1]. There is a characteristic delay in the onset of respiratory distress, approximately 6 to 12 days after the start of symptoms, which is somewhat atypical for other severe viral respiratory infections [2]. Several theories have been proposed for this delay, such as an indolent infection or viral persistence. However, data from viral cultures of severe acute respiratory syndrome coronavirus 2 (SARS-CoV-2) (not PCR) demonstrate a lack of positive cultures beyond day 9 of illness [3].

The timing of respiratory distress due to COVID-19 notably coincides with the onset of the humoral immune response, and there is evidence of autoantibodies among hospitalised COVID-19 patients, including anti-interferon and antiphospholipid antibodies [4, 5]. The pathogenicity of these autoantibodies is unclear. However, a pivotal study demonstrated high levels of extrafollicular B cell activation among severe cases of COVID-19 [6]. This type of immune response is characteristic of several autoimmune diseases, which raises the question whether severe COVID-19 is the result of a catastrophic autoimmune response that occurs among a subset of patients infected by the SARS-CoV-2 virus [7].

This study investigated the possibility that COVID-19 patients have autoimmune antibodies to annexin A2, a protective protein expressed in the lung and other organs. Since this phospholipid-binding protein is critical for fibrinolysis, lung elasticity, cell membrane repair, and integrity of the pulmonary vasculature, antagonism of annexin A2 may explain many of the hallmark clinical features of severe COVID-19 cases [8].

To evaluate this possibility, we analysed patient plasma on hospital day 0 or 1 among 86 patients at NYU Langone Health who were hospitalised for COVID-19 and confirmed to be positive by PCR. Anti-annexin A2 IgG antibodies were measured by ELISA. For comparison, we also studied IgG antibodies directed against annexin A5, which is another target of prothrombotic antiphospholipid antibodies, but is not known to have a direct role in maintaining the integrity of the pulmonary vasculature [9]. Antibody levels were calculated as relative units (RU) using a plasma sample previously identified as a high responder for IgG autoantibodies. Patients were categorised as 1) non-critical if hospitalised, but not intubated, 2) critically ill if hospitalised and intubated, or 3) died from COVID-19 during their hospitalisation.

After a descriptive analysis of study population, we analysed the anti-annexin A2 and A5 antibody levels as stratified by disease severity using ANOVA. To perform our primary analysis, we tested the association between antibody levels and death using multivariable logistic regression, adjusting for age, sex, race, and history of hypertension, diabetes and obesity (body mass index $>30 \mathrm{~kg} \cdot \mathrm{m}^{-2}$ ). A p-value of 0.025 was used to account for multiple comparisons. We used a margins analysis to graphically display mortality risk at a range of antibody levels. An analysis of outliers was performed to ensure that there were no extreme values for antibody levels that had an undue influence on the results. In addition, the robustness of the association between antibody levels and death was assessed with a sensitivity analysis that included the maximum laboratory values over the course of the hospitalisation for these COVID-19 patients. These commonly performed tests included white blood cell count (WBC), aspartate aminotransferase (AST),

Shareable abstract (@ERSpublications)

Levels of anti-annexin A2 antibodies at admission strongly predicted mortality among hospitalised COVID-19 patients. Given its critical protective function in the lung, annexin A2 antagonism may play an important role in the pathophysiology of COVID-19. https://bit.ly/2UMPr9w

Cite this article as: Zuniga M, Gomes C, Carsons SE, et al. Autoimmunity to annexin A2 predicts mortality among hospitalised COVID-19 patients. Eur Respir J 2021; 58: 2100918 [DOI: 10.1183/ 13993003.00918-2021]. 
alanine aminotransferase (ALT), creatine kinase (CK), lactate dehydrogenase (LDH), C-reactive protein (CRP), ferritin and D-dimer.

All statistical analyses were performed in Stata 16.2. Patients consented to use of their biospecimens for COVID-19 research through a central biorepository and protocol approved by the NYU institutional review board. A more detailed description of the methods can be accessed in a preprint of this study [10].

Of the 86 patients in our study, 28 were non-critical, 36 were critically ill and 22 died. Those who died had higher rates of hypertension $(p=0.04)$ and obesity $(p=0.05)$ when compared to patients who survived. In analysing the WBC, AST, ALT, CK, LDH, CRP, ferritin and D-dimer values among these hospitalised COVID-19 patients, the maximum values increased as expected by disease severity $(\mathrm{p}<0.01)$. We found higher average levels of anti-annexin A2 IgG antibodies among the hospitalised COVID-19 patients who died (1.16 RU, 95\% CI 0.95-1.37) when compared with the non-critical (0.80 RU, 95\% CI 0.66-0.94) and critically ill hospitalised COVID-19 patients (0.89 RU, 95\% CI 0.77-1.01). In comparison, there was no statistically significant difference in the average levels of anti-annexin A5 IgG antibodies when stratified by disease severity $(\mathrm{p}=0.32)$.

In our primary analysis of mortality among the 86 hospitalised COVID-19 patients, we found that anti-annexin A2 antibody levels strongly predicted death after adjustment for age, sex, race and comorbidities with an odds ratio of 9.3 per RU (95\% CI 1.9-44.6; p=0.005). In comparison, anti-annexin A5 antibody levels were not associated with a higher mortality rate (95\% CI 0.5-15.2; $\mathrm{p}=0.22$ ). Using a margins analysis, we graphically depicted predicted mortality rates across a range of levels for anti-annexin A2 antibodies (figure 1). In our sensitivity analysis, we added adjustments for the maximum WBC, AST, ALT, CK, LDH, CRP, ferritin and D-dimer levels. This multivariable regression demonstrated that anti-annexin A2 antibody levels strongly predicted mortality with an odds ratio of 12.9 per RU (95\% CI 1.5-108.7; $\mathrm{p}=0.019$ ) even after adjustment for the maximum laboratory abnormalities during the hospitalisation of these COVID-19 patients.

Our study finds evidence of higher levels of IgG antibodies directed against annexin A2 among COVID-19 patients who died. More importantly, anti-annexin A2 antibody levels strongly predicted mortality after controlling for patient risk factors and for the maximum levels of key laboratory markers associated with severe COVID-19.

Autopsy evidence demonstrates that severe cases of COVID-19 have extensive thrombotic disease, diffuse alveolar damage, and endothelial disruption that leads to pulmonary oedema and fibrin deposition [11]. These findings correlate with the clinical manifestations of severe COVID-19, which include diffuse

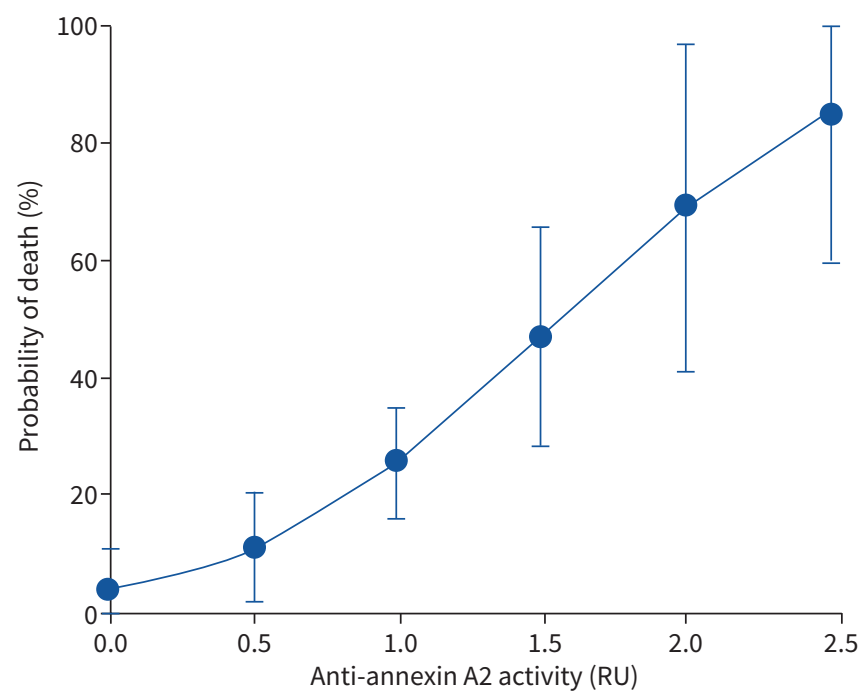

FIGURE 1 Prediction of mortality based on anti-annexin A2 antibody levels. Margins analysis based on logistic regression results depicts predicted mortality across a range of anti-annexin A2 antibody levels in relative units (RU). Error bars depict 95\% confidence intervals. 
clotting, acute respiratory distress syndrome (ARDS), non-cardiogenic pulmonary oedema, and fibrinous pulmonary exudates generally without bacterial superinfection [1]. Though our study does not present any direct evidence of the pathogenicity of these autoantibodies, antagonism of annexin A2 would explain many of the clinical findings that are characteristic of patients with severe COVID-19.

Annexin A2 is critical for fibrinolysis in the lung by acting as a co-receptor that activates endogenous tissue plasminogen activator to lyse clots and promote fibrin clearance [8]. More recently, annexin A2 was found to maintain the endothelial cell junctions in the lung microvasculature, preventing pulmonary oedema, especially in response to hypoxia [12]. Finally, annexin A2 promotes lung elasticity and is also involved cell membrane stabilisation and repair of pulmonary epithelial cells, thereby preventing apoptosis [13]. Its inhibition might also explain the diffuse alveolar damage, ARDS and pulmonary fibrosis seen in severe cases of COVID-19.

Notably, prior studies of SARS-CoV-1 identified autoantibodies that were cytotoxic to lung epithelial and endothelial cells and also specifically targeted annexin A2 among hospitalised SARS patients [14]. Loss of immune tolerance and a catastrophic autoimmune insult among a subset of patients could explain why certain patients develop severe symptoms of COVID-19 [15]. Other patients without this autoimmune response may have less severe symptoms, consistent with other coronavirus infections which only cause asymptomatic or mild disease.

While these autoantibodies could be non-specific markers of lung injury, it is critical to explore the pathogenicity of these anti-annexin A2 antibodies, as they could explain the underlying pathophysiology of severe COVID-19. Additional studies should assess the specificity of these autoantibodies to COVID-19 by investigating whether they also occur in other respiratory diseases such as influenza or other types of ARDS. Furthermore, we believe that the persistence of these anti-annexin A2 antibodies should be studied, especially among post-acute COVID-19 syndrome ("Long COVID”) patients with persistent respiratory symptoms. Marisol Zuniga ${ }^{1,8}$, Claudia Gomes
Qing Robert Miao ${ }^{5}$, David C. Lee ${ }^{6,7,9}$ and Ana Rodriguez
Q ${ }^{1,9}$

${ }^{1}$ Dept of Microbiology, NYU Grossman School of Medicine, New York, NY, USA. ${ }^{2}$ Division of Rheumatology, Dept of Medicine, NYU Long Island School of Medicine, New York, NY, USA. ${ }^{3}$ Division of Pulmonology and Critical Care Medicine, Dept of Medicine, NYU Long Island School of Medicine, New York, NY, USA. ${ }^{4}$ Dept of Pathology, NYU Grossman School of Medicine, New York, NY, USA. ${ }^{5}$ Dept of Foundations of Medicine, NYU Long Island School of Medicine, New York, NY, USA. ${ }^{6}$ Dept of Emergency Medicine, NYU Grossman School of Medicine, New York, NY, USA. ${ }^{7}$ Dept of Population Health, NYU Grossman School of Medicine, New York, NY, USA. ${ }^{8} \mathrm{M}$. Zuniga and C. Gomes contributed equally to this work. ${ }^{9}$ D.C. Lee and A. Rodriguez contributed equally to this article as lead authors and supervised the work.

Corresponding author: David C. Lee (david.lee@nyumc.org)

Acknowledgements: We want to specifically thank the Center for Biospecimen Research and Development at the NYU School of Medicine, Brian Fallon for bioinformatics support, and all of the volunteers that helped to obtain and process the samples used in this study and other research related to COVID-19.

Author contributions: A. Rodriguez and D.C. Lee designed the study. M. Zuniga and C. Gomez performed the experiments. A. Rodriguez and P. Cotiza collected the data and identified patient samples at NYU Langone Health. D.C. Lee drafted the manuscript and performed the statistical analyses. All authors interpreted the data and provided critical input to the manuscript.

Conflict of interest: None declared.

Support statement: This research was supported by an internal grant from the NYU Langone COVID-19 Special Fund. Funding information for this article has been deposited with the Crossref Funder Registry.

\section{References}

1 Wiersinga WJ, Rhodes A, Cheng AC, et al. Pathophysiology, transmission, diagnosis, and treatment of coronavirus disease 2019 (COVID-19): a review. JAMA 2020: 324: 782-793. 
2 Grant RA, Morales-Nebreda L, Markov NS, et al. Circuits between infected macrophages and T cells in SARS-CoV-2 pneumonia. Nature 2021: 590: 635-641.

3 Cevik M, Tate M, Lloyd O, et al. SARS-CoV-2, SARS-CoV, and MERS-CoV viral load dynamics, duration of viral shedding, and infectiousness: a systematic review and meta-analysis. Lancet Microbe 2021: 2: e13-e22.

4 Zuo Y, Estes SK, Ali RA, et al. Prothrombotic autoantibodies in serum from patients hospitalized with COVID-19. Sci Transl Med 2020; 12: eabd3876.

5 Bastard P, Rosen LB, Zhang Q, et al. Autoantibodies against type I IFNs in patients with life-threatening COVID-19. Science 2020: 370: eabd4585.

6 Woodruff MC, Ramonell RP, Nguyen DC, et al. Extrafollicular B cell responses correlate with neutralizing antibodies and morbidity in COVID-19. Nat Immunol 2020: 21: 1506-1516.

7 Zamvil SS, Hauser SL. Antigen presentation by B cells in multiple sclerosis. N Engl J Med 2021: 384: 378-381.

8 Hajjar KA. The biology of annexin A2: from vascular fibrinolysis to innate immunity. Trans Am Clin Climatol Assoc 2015: 126: 144-155.

9 Rand JH, Wu XX, Quinn AS, et al. The annexin A5-mediated pathogenic mechanism in the antiphospholipid syndrome: role in pregnancy losses and thrombosis. Lupus 2010: 19: 460-469.

10 Zuniga M, Gomes C, Carsons SE, et al. Autoimmunity to the lung protective phospholipid-binding protein Annexin a2 predicts mortality among hospitalized COVID-19 patients. medRxiv 2021; preprint [https://doi.org/ 10.1101/2020.12.28.20248807].

11 Rapkiewicz AV, Mai X, Carsons SE, et al. Megakaryocytes and platelet-fibrin thrombi characterize multi-organ thrombosis at autopsy in COVID-19: a case series. EClinicalMedicine 2020: 24: 100434

12 Luo M, Flood EC, Almeida D, et al. Annexin A2 supports pulmonary microvascular integrity by linking vascular endothelial cadherin and protein tyrosine phosphatases. J Exp Med 2017: 214: 2535-2545.

13 Dassah M, Almeida D, Hahn R, et al. Annexin A2 mediates secretion of collagen VI, pulmonary elasticity and apoptosis of bronchial epithelial cells. J Cell Sci 2014: 127: 828-844.

14 Fang YT, Lin CF, Liao PC, et al. Annexin A2 on lung epithelial cell surface is recognized by severe acute respiratory syndrome-associated coronavirus spike domain 2 antibodies. Mol Immunol 2010: 47: 1000-1009.

15 Theofilopoulos AN, Kono DH, Baccala R. The multiple pathways to autoimmunity. Nat Immunol 2017: 18: 716-724. 